\title{
关于电子转移引发的阴离子聚合产物 分子量分布的一种简单情况
}

\author{
颜 德 岳 \\ (同济大学化学系, 上海)
}

继 Nanda 等人 ${ }^{[1]}$ 处理了电子转移引发的阴离子型聚合反应产物的分子量分布问题之后, Peebles ${ }^{[2,3]}$ 又补充处理了同一反应体系的一种简单情况, 即瞬时引发的情况。本文作者 ${ }^{[4,5]}$ 业已 重新求解 Nanda 等人 [1]的反应动力学微分方程组, 得到分子量分布函数的严格解. 尽管 Peebles ${ }^{[2,3]}$ 采用了一些近似, 但在他的结果中，体系里无活性聚合物的分子量分布函数仍然包 含两个复杂的级数, 其中每一项都是一个不同的不完全 Gamma 函数. 这就给数值计算带来 困难. 本工作运用文献 [4] 所提供的方法, 重新处理 Peebles ${ }^{[2,3]}$ 提出的问题, 得到了最简单 的解析解,按照本文给出的种种数量关系,就可以由反应的初始条件和单体转化率计算产物的 各种分子参数.

在瞬时引发的情况下, Nanda 等人 ${ }^{[1]}$ 的动力学方程组可简化为

$$
\begin{aligned}
& d N_{2}^{* *} / d t=-2\left(k_{p}+k_{t}\right) M N_{2}^{* *} ; \\
& d N_{n}^{* *} / d t=2 k_{p} M N_{n-1}^{* *}-2\left(k_{p}+k_{t}\right) M N_{n}^{* *}, n>2 ; \\
& d N_{1}^{*} / d t=k_{t} M\left(2 \sum_{n=2}^{\infty} N_{n}^{* *}+\sum_{n=1}^{\infty} N_{n}^{*}\right)-\left(k_{p}+k_{t}\right) M N_{1}^{*} ; \\
& d N_{n}^{*} / d t=2 k_{t} M N_{n}^{* *}+k_{p} M N_{n-1}^{*}-\left(k_{p}+k_{t}\right) M N_{n}^{*}, n \geqslant 2 ; \\
& d N_{n}^{\prime} / d t=k_{t} M N_{n}^{*}, n \geqslant 1 .
\end{aligned}
$$

上列微分方程组的初始条件为 $\left.N_{2}^{* *}\right|_{t=0}=\frac{1}{2} I_{n},\left.N_{n>2}^{* *}\right|_{t=0}=\left.N_{n}^{*}\right|_{t=0}-\left.N_{n}^{\prime}\right|_{t=0}-0,\left.M\right|_{t=0}=$ $M_{0}$. 在 (1)一-(5) 及初始条件中的所有符号, 其意义同于文献 [4] 中的同一符号. 本文所处理 的反应体系还具有下列守恒条件:

$$
\begin{aligned}
& I_{0}-2 \sum_{n=2}^{\infty} N_{n}^{* *}+\sum_{n=1}^{\infty} N_{n}^{*} ; \\
& M_{0}-M=\sum_{n=2}^{\infty} n N_{n}^{* *}+\sum_{n=1}^{\infty} n\left(N_{n}^{*}+N_{n}^{\prime}\right) .
\end{aligned}
$$

为了方便起见, 把 $k_{t} / k_{p}$ 记为 $b$, 再令 $x=\int_{0}^{t} k_{p} M d t,(1)-(5)$ 就可变换为常系数线性微分 方程组. 由此可得

$$
N_{n}^{* *}=\frac{I_{0}}{2} \frac{(2 x)^{n-2} e^{\left.-x_{1}+b\right) x}}{(n-2) !}
$$

本文 1981 年 3 月 31 日收到。 


$$
\begin{aligned}
N_{*}^{*}= & \frac{I_{0} b e^{-(1+b) x}}{(1+b)^{n}}\left\{(1+b) e^{-(1+b) x} \sum_{i=n-1}^{\infty} \frac{[2(1+b) x]^{i}}{i !}\right. \\
& \left.-b \sum_{i=n-1}^{\infty} \frac{[(1+b) x]^{i}}{i !}-\frac{[(1+b) x]^{n-1}}{(n-1) !}\right\} ; \\
N_{n}^{\prime}= & \frac{I_{0} b^{2} e^{-(1+b) x}}{(1+b)^{n}}\left\{\left[(1+b) x-\frac{n-1}{2}\right] e^{-(1+b) x} \sum_{i=n-1}^{\infty} \frac{[2(1+b) x]^{i}}{i !}\right. \\
& +\frac{[2(1+b) x]^{n-1} e^{-(1+b) x}}{2(n-2) !}-\left(b x+1-\frac{n b}{+b}\right) \sum_{i=n-1}^{\infty} \\
& \left.\times \frac{[(1+b) x]^{i}}{i !}+\left(1-\frac{n b}{1+b}\right) \frac{[(1+b) x]^{n-1}}{(n-1) !}\right\} ; \\
N_{n}= & I_{c_{0} e^{-(1+b) x}}\left\{\left[(1+b)(1+b x)-\frac{b(n-1)}{2}\right] e^{-(1+b) x} \sum_{i=n-1}^{\infty}\right. \\
& (1+b)^{n}\left[(1+b]^{n-2} e^{-(1+b) x}\right. \\
& \times \frac{[2(1+b) x]^{i}}{i !}+(1+b)\left(1+b+2 b^{2} x\right) \frac{[2(1+b) x}{2(n-2) !} \\
& -b^{2}\left(b x+2-\frac{n b}{1+b}\right) \sum_{i=n-1}^{\infty} \frac{[(1+b) x]^{i}}{i !} \\
& \left.+b\left(b-1-\frac{n b^{2}}{1+b}\right) \frac{[(1+b) x]^{n-1}}{(n-1) !}\right\} .
\end{aligned}
$$

上面的 (11) 式是体系中生成的全部摮合物的分子量分布函数,亦即

$$
N_{n}-N_{*}^{* *}+N_{*}^{*}+N_{n}^{\prime} \text { 。 }
$$

体系中生成的全部聚合物的数均及重均聚合度如下:

$$
\begin{aligned}
& \bar{P}_{s}-\frac{2[1+(1+b) x]}{1+2 b x} ; \\
& \bar{P}_{-}-\frac{2+\left(b+3+\frac{2}{b}\right) x-\frac{1}{b} x e^{-2 b x}-\frac{1}{2 b^{2}}\left(1-e^{-2 b x}\right)+\frac{1}{b}\left(1-e^{-b x}\right)}{1+(1+b) x} .
\end{aligned}
$$

在反应中生成的双阴离子聚合物、单阴离子聚合物及无活性愁合物的克分子分数分别为

$$
\begin{aligned}
& f_{* *}^{* *} \frac{e^{-2 b x}}{1+2 b x} ; \\
& f_{*}^{*}=\frac{2\left(1-e^{-2 b x}\right)}{1+2 b x} ; \\
& f_{*}^{\prime}=\frac{2 b x-\left(1-e^{-2 b x}\right)}{1+2 b x}
\end{aligned}
$$

显然 (15)-(17) 是这一反应体系的官能度分布公式,而平均官能度则为

$$
\bar{f}=\frac{2}{1+2 b x} \text {. }
$$

在上面所得各种分子参数的表达式中,参变量皆为 $x$, 后者可由下式确定:

$$
x=\frac{\frac{M_{0}}{I_{0}} y-1}{1+b},
$$

这里 $y-\frac{M_{0}-M}{M_{0}}$ 为单体转化率. 若将 (19) 代人 (13) 和 (18), 则可得 


$$
\widetilde{P}_{n}=\frac{2(1+b) y M_{0}}{(1-b) I_{0}+2 b y M_{0}} ; \vec{f}=\frac{2(1+b) I_{0}}{(1-b) I_{0}+2 b y M_{0}} .
$$

由此可见, 数均聚合度或平均官能度与单体转化率、引发剂和单体的初始浓度之间存在简单的 数量关系. 对于其它分子参数亦可进行类似的计算,只是运算过程要复杂一些. 当 $b-0.01$,

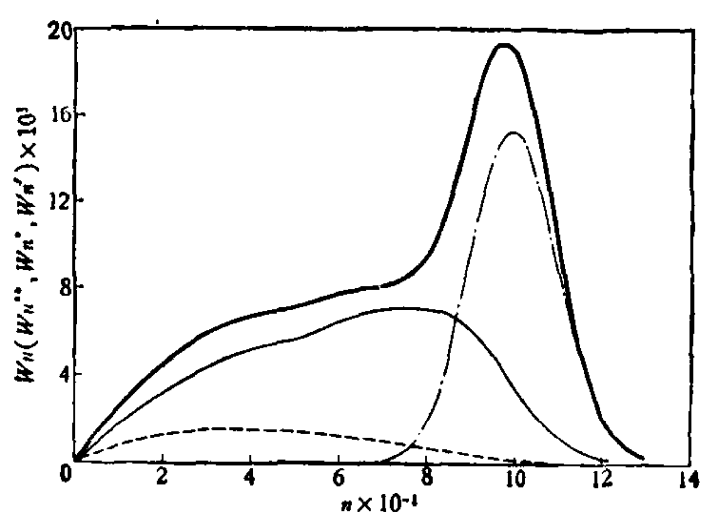

图 1 不同官能度藂合物及全部 聚合物的分子量分布曲线 $h=0.01, I_{0} / M_{0}=0.001, y=5 \% ;$ 一一双 阴度子䈋合物；一单阴离子慗合物；一无活性 聚合物；一全部聚合物

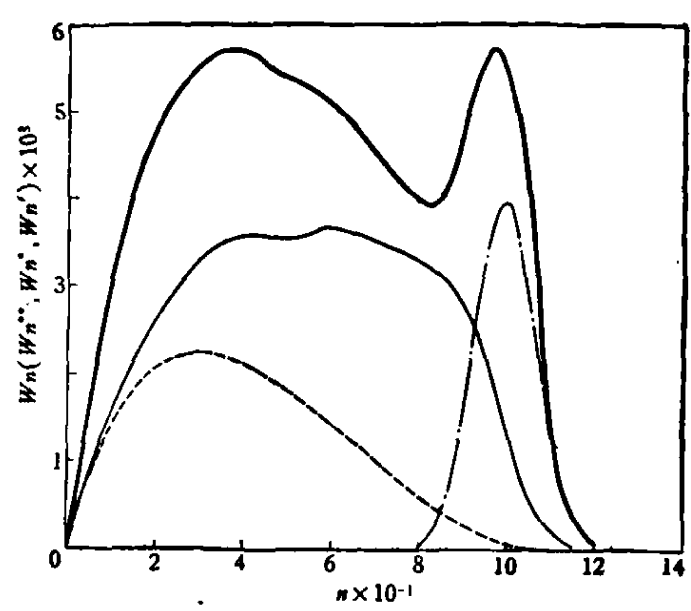

图 2 不同官能度聚合物及全部聚合物的分 子晴分布曲线 $y=10 \%$, 其余同图 1

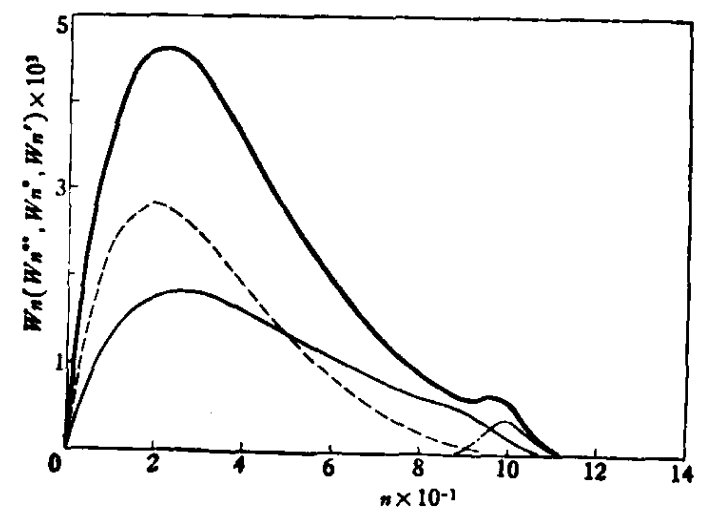

图 3 不同官能度笔合物及全部㲠合物的分子量分布曲线 $y=20 \%$, 其余同图 1

$I_{0} / M_{0}=0.001$ 时,体系中双阴离子聚合物、单阴离子聚合物、无活性聚合物及全部聚合物的分 子量分布随单体转化率的变化见图 1-3. 图中 $W_{s}^{* *}=n N_{n}^{* *} / \sum_{n} n N_{n}, W_{n}^{*}=n N_{n}^{*} / \sum_{n} n N_{n}$, $W_{n}^{\prime}=n N_{n}^{\prime} / \sum_{n} n N_{n}, W_{n}=n N_{n} / \sum_{n} n N_{n}$

\section{* 妾文}

11 I Nanda, V. S. \& Jain, R. K., Trans. Faraday Soc., 64 (1968), 1022.

[2] Peebles, Jr. L. H., J. Polymer Sci., A2 (1970), 8:1235.

[3] Peebles, Jr. L. H., MWD in Polymers, Interscience, New York, 1971, 178.

[4]硕德岳,高分子通讯, 1980, 5:271.

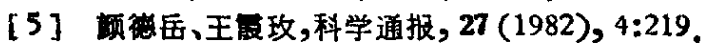

